\title{
Oncogene N-RAS
}

National Cancer Institute

\section{Source}

National Cancer Institute. Oncogene N-RAS. NCI Thesaurus. Code C16889.

Human Oncogene N-RAS is a mutated variant of NRAS Gene (RAS Family), which encodes p21 N-Ras Protein, a monomeric GT Pase involved in transmembrane signal transduction that alternates between inactive GDP-bound and active GT P-bound forms. RAS is activated by a guanine nucleotide-exchang e factor and inactivated by a GTPaseactivating protein. Mitogen-stimulated RAS stabilizes MYC protein and enhances MYC accumulation by the RAS/RAF/MAPK pathway, which appears to inhibit the proteasomedependent degradation of MYC. Implicated in a variety of human tumors, specific amino acid mutations activate c-RAS and transform cells. Oncogene NRAS disrupts normal cell function. 